Pacific Journal of Mathematics

EXTENSIONALITY AND CHOICE IN CONSTRUCTIVE 


\title{
EXTENSIONALITY AND CHOICE IN CONSTRUCTIVE MATHEMATICS
}

\author{
Michael BeEson
}

\begin{abstract}
We study the relationships between two kinds of formal systems which have been proposed for formalizing modern constructive mathematics. Essentially, we show how to interpret the set-theoretic systems of Friedman and Myhill in the operation-theoretic systems of Feferman. As a by-product of this interpretation, we prove that Friedman's system $\underline{B}$ and certain of Feferman's systems are conservative over intuitionistic arithmetic. We also hope the interpretation casts some light on the nature of the concepts axiomatized in the two systems.
\end{abstract}

We now explain more leisurely the background of the results and methods of this paper. Ten years ago, Bishop published a book showing that vast portions of modern analysis can be systematically constructivized. His methods involved only careful definitions of the mathematical concepts involved, and a restriction that no proof by contradiction be allowed. That is, he departed from the older school of intuitionism, which introduced such nonclassical ideas as choice sequences, and along with them, some axioms which are not classically valid. Because of this, each of Bishop's theorems is classically valid; this has made the "new constructivism" considerably more appealing to mathematicians than its predecessors. It was, however, a challenge to logicians to find suitable formal systems in which the work of the new constructivists could be carried out. Though Bishop's book is entitled Foundations of Constructive Analysis, the title is appropriate only in one sense of the word "foundations"; a thorough philosophical analysis of the conceptions underlying constructivity is still lacking. The same, of course, is more or less true of classical set theory. Several logicians set out to do for the new constructivism what Zermelo, Frankel, and Russell did for classical mathematics; namely, to give formal systems encompassing all the usual arguments, and based on some rough intuition of the underlying ideas. These formal systems should in turn serve to sharpen our understanding of the foundations of the subject.

There has not yet emerged a single formal system for the new constructivism which is as universally recognized as adequate for its purpose as is $Z F$ for classical set theory. There are still several different approaches being discussed. Two approaches which have received the most attention are those of Feferman and Friedman. In order to say more, and especially in order to explain the title of 
the paper, it is necessary to describe the systems of Feferman and Friedman briefly.

Friedman's systems are based on set theory; they are like $Z F$ except that (i) the power set axiom is replaced by the axiom of "exponentiation", which says the set of functions from $A$ to $B$ exists, if $A$ and $B$ are sets, (ii) the axiom of dependent choices is added, but not the full axiom of choice, (iii) a restricted form of induction is used; there are also other differences from $Z F$. In these systems, one formalizes constructive mathematics just as one does classical mathematics; for instance, functions are to be identified with their graphs. Thus a real number is a certain set of pairs $\langle n, r\rangle$ with $r$ rational. Or, if you please, an equivalence class of such sets, just as in standard analysis texts. Friedman's systems include the axiom of extensionality.

Feferman's systems seem to be quite different. They are based on theories of "operations" which can apply to other constructive objects, perhaps themselves. These operations may sometimes be undefined. The variables of Feferman's systems are to range over "the constructive universe", which includes the operations and also the "classifications", which are rather like sets. For example, there is a classification $N$ of natural numbers; each number is identified with a certain operation. Then there is the classification of rational numbers, and then the real numbers, which are certain sequences of rationals, i.e., operations from $N$ to the rationals. There is a comprehension axiom to guarantee the existence of enough classifications, and some combinatorial axioms to guarantee the existence of enough operations.

The two approaches to formalizing Bishop's work seem at first to be incommensurable, because of the following difficulties:

(1) The axiom of extensionality for classifications is inconsistent with certain versions of Feferman's theories, and possibly inconsistent with others.

(2) If one wishes to show that a function (in Feferman's sense) is the same as a function (in Friedman's sense, as a graph), in one direction it is easy: we can always form the graph of an operation. But given a set $X$ such that $\forall z \in A \exists ! w \in B\langle z, w\rangle \in X$, we do not know if there is an operation $f$ which produces $w$ from $z$. To get $f$, we would need a version of the axiom of choice. Unfortunately, the version we need is too strong - it is inconsistent with Feferman's theory, and implies the law of the excluded middle in Friedman's theory.

Difficulty (1) can be overcome by the simple expedient of dropping 
extensionality, as mentioned above (but see also footnote 1 for a discussion of extensionality in Feferman's systems). Metamathematically, there is a problem about whether extensionality enables us to prove any more arithmetical theorems; but we have solved this problem.

Difficulty (2) is more fundamental. To explain the solution, let me now describe the system $F$ : it consists of Feferman's system, plus the axiom of dependent choices $D C$. Since $D C$ is constructively valid, we have a single system, encompassing both Feferman's and Friedman's approaches to the formalization of constructive mathematics (modulo some other points discussed below). Using $D C$, we can prove the equivalence of functions-as-operations and functions-as-graphs, if the domain of the function is countable. In an appendix, we give reasons why this is adequate for the practice of mathematics; roughly speaking, it is because only continuous functions arise in mathematical practice.

From the metamathematical point of view, the main purpose of this paper is to prove that $F, \underline{B}$, and $H A$ all have the same arithmetical theorems, a result which itself has some foundational significance. In order to say more, we outline the four steps of the proof:

Step 1 . Show that extensionality can be deleted from $\underline{B}$ without loss of arithmetical theorems.

Step 2. Interpret the rest of $\underline{B}$ in a suitable theory in Feferman's language, namely a weak theory $F_{0}$ plus a certain unusual axiom of choice.

Step 3. Show that the axiom of choice in this theory can be eliminated without loss of arithmetical theorems, thus reducing $B$ to the weak theory $F_{0}$.

Step 4. Show that $F_{0}$ is conservative over $H A$.

Steps 1 and 4 are relatively easy; Step 1 is done in [3], by interpreting sets as sets of rank $\langle\omega+\omega$, with suitable defined equivalence

${ }^{1}$ It is an interesting question whether extensionality can be consistently added to Feferman's theories. In [9], Feferman showed that extensionality is inconsistent with the versions of his theories considered there. In [4], however, reasons are given for not including decidable equality as an axiom; and Feferman's inconsistency proof depends heavily on decidable equality. The versions of Feferman's theories considered here have decidable equality only for integers; it is open whether extensionality can be consistently added.

Certainly one cannot simply read the axioms of $\underline{B}$ as being about classifications and hope that $\underline{B}+F_{0}$ would be consistent: By elementary comprehension, form the universe $V=\{x: x=x\}$. Then apply $\Delta_{0}$-comprehension to form the Russell set $\{x \in V: x \notin x\}$. This shows quite clearly the difference between classifications and sets, which is exploited, or rather analyzed, in Lemma 2 of $\S 2$. This lemma shows that in some sense $F+B+$ CT-ext can be "contained in" a consistent system. 
relations. Step 4 is a simple Kripke-model argument. In case we only want to re-prove Friedman's result that $B$ is conservative over classical arithmetic, Step 3 is also simple: to reduce $B$ to $F_{0}+$ classical logic (which is conservative over $P A$ ). For the conservativeness over $H A$, however, we need a "Goodman-style" theorem. This is proved in [2]. Step 2 presents formidable difficulties. For instance, to interpret the exponentiation axiom of $B$, which says the set of functions from $A$ to $D$ exists, if $A$ and $D$ are sets, we need to be able to prove the equivalence of functions-as-operations and functions-asgraphs. For this we seem to need an axiom of choice which is inconsistent with $F$. The means of solving this difficulty is to use instead an unusual axiom of choice, weaker than the one which first comes to mind, which is consistent. Even after overcoming this difficulty, we still have another difficulty interpreting the $\Delta_{0}$ separation axiom of $B$. This difficulty is quite interesting, foundationally, because to overcome it, we must explain the notion of "set" axiomatized in $\underline{B}$ in terms of the notion of "classification" axiomatized in $F$. Whatever the foundational merits of our answer, it suffices for the metamathematics. It is this: a "set" $X$ is a classification such that the structure $(T C(X), \varepsilon)$ is given by a classification, where $T C(X)$ is the transitive closure of $X$. In other words, before we call a classification a set, we must know the whole (hereditary) membership structure of it. Thus, the universe $V$ is a classification, but not a set.

This discussion is the starting point of a project which lies beyond the scope of this paper: to analyze the different possible constructive notions of "set". We return briefly to this idea in $\S 3$, in connection with a discussion of axioms of choice.

The paper is organized as follows: In $\S 0$, we describe the theories we shall consider. In $\S 1$, we discuss informally the relationship between constructive mathematical practice as formalized in Feferman's systems and as formalized in Friedman's systems. In $\S 2$, we carry out the proof of our conservative extension result. In $\S 3$, we summarize a number of known results on axioms of choice.

o. Preliminaries. We assume familiarity with intuitionistic arithmetic $H A$. We proceed to describe Friedman's and Feferman's theories. Friedman's theory $B$ is formulated in a two-sorted language, with one sort of variables for numbers and one sort for sets. There are two membership relations, for numbers belonging to sets and sets belonging to sets. There is a successor function symbol from integers to integers (but plus and times are to be set-theoretically defined). The axioms of $\bar{B}$ are as follows; if they are not written out, they are exactly as usual for classical set theory. Also, ordered pairs, 
functions, etc. are as usual.

1. Pairing

2. Union

3. Infinity. $\exists x \forall n(n \in x)$

4. Extensionality. $\forall x(a \in q \leftrightarrow b \in x) \rightarrow a=b$

5. $\Delta_{0}$-separation. $\exists z \forall x(x \in z \leftrightarrow x \in a$ and $\phi(x))$, for $\phi$ a $\Delta_{0}$ formula, i.e., having only bounded quantifiers.

6. Dependent choice. $\forall x \in a \exists y \in a(\langle x, y\rangle \in X) \rightarrow \forall x \in a \exists f \in a^{\omega}$

$$
(f(0) \in x \quad \& \quad \forall n \in \omega(\langle f(0), f(n+1)\rangle \in X) .
$$

7. Exponentiation. $\exists X \forall f(f \in X \leftrightarrow F c n(f)$ and $\operatorname{Dom}(f)=A$ and Rng $(f) \subseteq B)$. Here $F c n(f)$ is $\forall a, b, c(\langle a, b\rangle \in f$ and $\langle a, c\rangle \in f \rightarrow b=c)$.

8. Abstraction. This is a weak consequence of replacement, which asserts that $\{\{x \in a: \phi(x, y)\}: y \in a\}$ exists, if $\phi$ is a $\Delta_{0}$ formula.

9. Induction (on the integers). More precisely, "restricted induction" on the integers.

$$
0 \in X \quad \& \quad \forall n(n \in X \longrightarrow n+1 \in X) \longrightarrow \forall n(n \in X) .
$$

The help the reader, we mention some axioms which are not in $B$. For instance, full induction on the integers whould have an arbitrary formula in place of $X$ in axiom 9 ; this is one source of the proof-theoretic weakness of $\underline{B}$. The power set axiom is not in $\underline{B}$; constructively, the exponentiation axiom is considerably weaker, though classically equivalent. It was Myhill who discovered that exponentiation suffices for formalizing mathematical practice [12]; it was Friedman [11] who noticed the results of restricting induction. The full axiom of choice is not included in $\underline{B}$; in fact, it implies the law of the excluded middle. (See $\S 3$ for more discussion of axioms of choice.) The foundation axiom, and its cousin, transfinite induction, are not in $\underline{B}$. In [11] and [3], a variety of intuitionistic set theories is considered, drawing from these axioms; but in this paper, we consider only $\underline{B}$.

We do, however, have need to consider the theory obtained by dropping extensionality from $\underline{B}$. We denote this theory by $\underline{B}$-ext, but it is not quite as simple as just dropping the axiom of extensionality. First, we definitely include equality in the language (in the case of extensional $\underline{B}$, it can be completely eliminated, substituting extensional equality in the equality axioms.) Second, we include a system of terms to denote the sets which are asserted to exist by some of the axioms. Third, we formulate the axioms of exponentiation and abstraction carefully, choosing from among several formulations which are equivalent when extensionality is present. We now give these modified axioms and the system of terms to be included. 
The modified exponentiation axiom is as follows: Let $F c n(f)$ be

$$
\begin{aligned}
\forall x, y, z(\langle x, z\rangle \in f \quad \& \quad & \langle x, y\rangle \in f \longrightarrow \forall u(y \in u \longleftrightarrow z \in u)) \\
& \& \quad \forall a \in f \exists b, c(a=\langle b, c\rangle) .
\end{aligned}
$$

Let $\operatorname{Dom}(f)=a$ and Rng $(f) \subseteq b$ abbreviate $\forall x \in a \exists y \in b(\langle x, y\rangle \in f)$ and $\forall\langle x, y\rangle \in f(x \in a$ and $y \in b)$. Then the exponentiation axiom says $\exists X \forall g(F c n(g) \quad \& \quad \operatorname{Dom}(g)=A \quad \& \quad \mathrm{Rng}(g)=B \rightarrow \exists f \in X \forall x \in A(f(x)=$ $g(x)))$.

REMARKS. The choice of this version of the exponentiation axiom is governed by the following considerations: (i) It should be strong enough to interpret the exponentiation axiom of $B$ under the interpretation given in [3]. For this reason one uses extensional equality in the definition of a function, so that functions defined with values equal to the equivalence classes of some equivalence relation are legitimate functions; or to put it another way, so that one can construct the set of functions in Bishop's sense from $(A, E)$ to $(B, R)$, where $E$ and $R$ are equivalence relations giving the "equality" relations of sets $A$ and $B$. (ii) It should be weak enough that it can be interpreted in Feferman's theories as we shall do in this paper. For that reason we cannot require that the set of all functions from $A$ to $B$ exists, only that some set containing one function with the same values as any given function from $A$ to $B$ exists. There is a third consideration, not relevant to this paper: (iii) It should be soundly interpreted under realizability and forcing as in [3]. Requirement (iii) would be met if we took the axiom of $\underline{B}$, without the modification mentioned under (ii) (but using extensional equality in the definition of $F c n$ ); it is also met with the axiom as given.

The modified abstraction axiom asserts the existence of a set $X$, such that for $y \in a$, there is $w$ in $X$ with $x \in w \leftrightarrow(x \in a \& \phi(x, y))$, and for each $w$ in $X$ there is $y$ in $A$ with the same formula true.

We now specify the exact system of terms to be included in our nonextensional set theories; these terms are built up from the following constants and function symbols. We also give the defining axioms for these symbols.

(i ) a constant symbol $\phi$, and the axiom $\forall x(x \notin \phi)$.

(ii) a function symbol \{\} , and the axiom $z \in\{x, y\} \leftrightarrow z=x \vee z=y$.

(iii) a function symbol for union, and the axiom

$$
y \in \bigcup_{z \in A} z \longleftrightarrow \exists z \in a(y \in z) .
$$

Then $a \cup b$ abbreviates $\bigcup_{z \in\{a, b\}} z$

(iv) a constant symbol $\omega$ and axiom 


$$
\phi \in \omega \quad \& \quad \forall z(z \in \omega \longrightarrow z \cup\{z\} \in \omega)
$$

and

$$
\forall x(\phi \in X \& \forall z(z \in X \longrightarrow z \cup\{z\} \in X) \longrightarrow \omega \leqq X)
$$

(v) For each formula $P$ for which separation is allowed, a function symbol $\{x \in a: P(x, a)\}$ and the obvious axiom.

(vi) Symbols for dependent choice: if $P$ is a formula for which dependent choice is allowed, we have a function symbol $i_{p}$ with the axiom $\forall x \in \omega \exists ! y \in \omega P(x, y) \& x_{0} \in \omega \rightarrow i_{p}\left(x_{0}\right) \in \omega^{\omega} \& i_{p}\left(x_{0}\right)(0)=x_{0}$ and $\forall n \in \omega P\left(i_{p}\left(x_{0}\right)(n), i_{p}\left(x_{0}\right)(n+1)\right)$.

Note that we include choice symbols only for functions from $\omega$ to $\omega$. Thus at least we have terms for all the primitive recursive functions, so that our nonextensional theories contain arithmetic in a natural sense. Note that there are no terms corresponding to the collection axiom. Generally speaking, it seems that we get several theories of different strength by including or not including constants and functions symbols corresponding to the various axioms. We certainly need to include separation terms in order to achieve the technical results we want; the rest seem to be optional.

The above description requires a little elaboration, since the formula $P$ in a term $\{x \in a: P(x)\}$ may itself contain other terms. One way to make our definitions completely precise is as follows: Start by adding a list $f_{n}$ of function symbols to the language (for the separation terms) and similar lists for the other types of terms required. Then Gödel number all the formulae of the language, and then write $\{x \in a: P(x, y)\}$ for $f_{n}(a, y)$, where $P$ has Gödel number $n$. Of course, we now have more terms than we want, since we only want such terms for certain formulae $P$. One can either delete the extra terms from the language, or leave them in, but add no axioms about them. To specify which formulae $P$ are allowed, for example in the case of $\Delta_{0}$-separation, we add a clause to the definition of a $\Delta_{0}$ formula specifying that if terms $\{x \in b: Q(x)\}$ occur in the component formulae, then $Q$ is already $\Delta_{0}$, and similarly for abstraction and choice terms occurring in the component formulae. Note that generally when we add more symbols to the language, there are more $\Delta_{0}$-formulae.

Now we describe the theory $F_{0}$ of Feferman. This theory has variables ranging over the constructive universe. There is a relation $\operatorname{App}(f, x, y)$, which we abbreviate $f(x) \cong y$, and a relation $\mathrm{Cl}(x)$, which means $x$ is a classification, or species. There are constants $k, s, \underline{0}, d$, 
$\boldsymbol{p}, \boldsymbol{p}_{1}, \boldsymbol{p}_{2}$. The last three are for pairing and unpairing functions, $\boldsymbol{d}$ is for a definition-by-integer-cases function, 0 is for zero, and $\underline{k}$ and $s$ are the usual combinators given by $k x y \cong x$ and $s x y z \cong x z(y z)$. There are also some constants for classification, namely $N$ (for the integers) and $c_{n}$ for $n=0,1,2, \cdots$. We write $c_{B}$ for $c_{n}$ if $n$ is the Gödel number of $B ; c_{B}(y)$ is supposed to denote $\{x: B(x, y)\}$ for certain formulae $B$. In order to state the axioms, we introduce the idea of an application term, or for short, a term. Each constant or variable is a term, and if $t$ and $s$ are terms, so is $t(s)$. (But we emphasize that these terms are not part of the official language.) We use the following abbreviations in connection with application terms:

$$
\begin{array}{ll}
t_{1} t_{2} \cdots t_{n} & \text { for }\left(\cdots\left(t_{1} t_{2}\right) \cdots\right) t_{n} \text { (association to the left) } \\
t \cong y & \text { for } t=y \text { when } t \text { is a variable or constant } \\
t s \cong \mathrm{y} & \text { for } \exists x \exists z(t \cong x \& s=z \& \operatorname{App}(x, z, y)) \\
t \downarrow & \text { for } \exists y(t \cong y) \\
t \cong s & \text { for } \forall y(t \cong y \longleftrightarrow s \cong y) \\
\phi(t, \cdots) & \text { for } \exists y(t \cong y) \& \forall y(t \cong y \longrightarrow \phi(y, \cdots))
\end{array}
$$

We now can give the axioms of $F_{0}$. These are in three groups, the combinatorial axioms, the comprehension axiom, and (restricted) induction.

Combinatorial axioms.

(i) $\operatorname{App}(f, x, y) \& \operatorname{App}(f, x, w) \rightarrow y=w$

(ii) $s x y \downarrow \& s x y z \cong x z(y z)$

(iii) $k x y \cong x$

(iv) $p x y \downarrow \& p_{1} z \downarrow \& p_{2} z \downarrow \& p_{1}(p x y) \cong x \& p_{2}(p x y) \cong y$

(v) $7(\boldsymbol{p} x y \cong 0)$

(vii) $\forall x, y \in N((x=y \rightarrow d a b x y \cong a) \&(x \notin y \rightarrow d a b x y \cong b))$.

Comprehension axiom. First we have the simple axiom $x \in y \rightarrow$ $\mathrm{Cl}(y)$. Then we have $\exists X\left(\forall x(x \in X \leftrightarrow \phi(x, y, W)) \& X \cong c_{\phi}(y, W)\right)$. Here $\phi$ is restricted to be an elementary formula. The definition of an elementary formula, and the convention governing the use of capital letter variables, is as follows: When we write a capital letter, we mean that this variable is restricted to $C l$. Thus $\forall X$ abbreviates $\forall x(\mathrm{Cl}(x)) \rightarrow$. When we write $\phi(X)$, we mean $\mathrm{Cl}(x) \rightarrow \phi(x)$. This applies to every formula containing $X$; so for instance the comprehension axiom above has $\mathrm{Cl}(W)$ implicitly in front. Now we define an elementary formula $\phi$ by:

(i) $\phi$ does not mention Cl.

(ii) If $\phi$ contains an atomic subformula $s \in t$, then $t$ is one of 
the free variables $X$ of $\phi$.

(iii) $\phi$ does not contain any constants $c_{B}$. This is an inessential restriction, because we can form $\{x: \phi(y, W)\}$ and then substitute a constant for $W$ or $y$.

Induction. $F_{0}$ has "restricted induction":

$$
\underline{0} \in X \quad \& \quad \forall n \in N\left(n \in X \longrightarrow n^{\prime} \in X\right) \longrightarrow \forall n \in N(n \in X) .
$$

(Here $n^{\prime}$ is $p(n, \underline{0})$.) This is called "restricted induction" as opposed to "full induction" in which $X$ is replaced by an arbitrary formula. This is the last axiom of $F_{0}$.

The theory $F . \quad F$ consists of $F_{0}$ together with the axiom of dependent choices $D C$, written just as in the list of axioms of $\boldsymbol{B}$.

Church's thesis. This axiom asserts that every number-theoretic function is recursive.

$$
\forall f \in N^{N} \exists e \in N \forall x \in N(f(x)=\{e\}(x)) .
$$

We shall have occasion to consider the axiom of choice in several forms. One of them is

AC: $\quad \forall a \in X \exists b \in Y\langle a, b\rangle \in W \longrightarrow \exists f \in Y^{X} \forall a \in X(\langle a, f(a)\rangle \in W)$.

The choice function $f$ is not required to be extensional, if the axiom is considered in Feferman's theories; it is just an operation. Nevertheless, the axiom is inconsistent with $F_{0}$ (see $\S 3$ ). It is, however, consistent with a theory $F_{0}^{-}$which is obtained by restricting the comprehension axiom of $F_{0}$ to elementary formulae which are "almostnegative". An "almost-negative" formula is a formula $\phi(t)$, where $\phi$ is negative and $t$ is (a list of) application terms. (So when $\phi$ is written out, there will be some existential quantifiers used to say $t$ is defined.)

REMARKS. The terminology for the many theories in Feferman's language can be confusing, especially since the first-considered (and hence first-named) theories have stronger axioms than the weak theories on which attention has since focussed. If we add full induction to $F_{0}^{-}$, we get was called $B E M$ ("basic explicit mathematics") in [5] and [6]. The theory $B E M+C A$ is $F_{0}+$ full induction. If we add the join axiom, we get $E M N$ and $E M N+C A$, in the terminology of [4]; $E M N$ was the weakest theory explicitly studied there. (This was before the realization that join is not needed for formalizing mathematical practice.) If we then add axioms for transfinite induction, we get $E M$ and $E M+C A$ ( $E M$ for "explicit mathematics"). If 
we add decidable equality and full definition by cases, we get $T_{0}^{-}$(with only almost-negative comprehension) and $T_{0}$ (with full elementary comprehension); these were the theories originally introduced in [9]. (The theory $T_{1}$ of [9] is not a constructive theory and is not relevant here.)

1. Formalizing constructive mathematics. In this section we shall discuss Friedman's and Feferman's different proposals for formalizing informal constructive mathematics. We begin with Friedman's approach. To each informal assertion $A$ of constructive mathematics, we can assign a formalization $A_{H}$ of $A$ in the language of set theory; given the idea of regarding a function as identical with its graph, we may say that $A_{H}$ is a "natural" formalization of $A$. The point here is that the fundamental constructive notion of operation or construction is never needed in actual practice, because all we ever use about an operation is its values. After this fundamental observation, the next step is to analyze what principles are actually used in mathematical practice, to show that $B$ suffices to prove $A_{H}$ for each informal theorem. (Since of course Gödel's theorem will take us beyond any formal system, what we mean is something like this: $\underline{B}$ proves $A_{H}$ for any $A$ occurring in Bishop's book, or which might naturally have occurred in Bishop's book.) This analysis has been carried out by Friedman; it consists mainly in checking that we do not need power set, exponentiation suffices; and we never use full induction, restricted induction suffices.

Carrying this analysis further, we maintain that extensionality and union can also be dropped. First we discuss extensionality. In Bishop's book, extensionality is never used. Of course, we require that functions preserve the "equality" relations on their domains and ranges, but these relations are only equivalence relations - the axiom of extensionality is irrelevant. Indeed, in the formal development of mathematics in $B$, the axiom of extensionality is used only for regarding reals as equivalence classes of Cauchy sequences. In the first place, this is a deviation from constructive mathematical practice: as Bishop remarked, the classical mathematician puts things in equivalence classes only to take them out again. In the second place, one can, if one wishes, use equivalence classes as reals, then defining an equality relation between such classes. In the absence of extensionality, this will not be literal equality, but no matter; so extensionality is not necessary even if we want to regard reals as equivalence classes. As a matter of fact, the presence of extensionality in $\underline{B}$ is a sales device, designed to make constructive mathematics more palatable to the classical mathematicians. One should not take this purpose lightly but the present point is that extensionality is irrelevant to mathe- 
matical practice ${ }^{1}$.

Since Feferman's theory has a membership relation, we can regard the formula $A_{I I}$ of set theory also as a formula of Feferman's system. We shall take an interest in the extent to which Feferman's theories are adequate to prove $A_{H}$ for informal theorems $A$. We mention this now, because we intend next to discuss the role of the union axiom in formalizing constructive mathematics, and the discussion applies to both Feferman's and Friedman's systems, since one can formulate a union-like axiom in either system. At first it appears that we need such an axiom, since we often want to form $\mathrm{U}_{x \in S} A_{x}$, or, more explicitly, $\{\langle y, x\rangle: y \in x$ and $x \in S\}$. In other words, we want the union of a family $S$. I think Feferman was the first to suggest that in practice, usually $S$ is already "pre-joined". For instance, if $S$ has a transitive closure, then the union above can be formed by the separation axiom alone. (Proving that every set has a transitive closure is not possible in $\boldsymbol{B}$.) If one looks at the place in Bishop's book where he talks about taking the union of a family, one finds the definition of a family sufficiently loose that it could be interpreted to mean "pre-joined family", which indicates that union can be replaced by separation. An examination of the book bears out this impression. Feferman has also checked the Bishop-Cheng notes on measure theory, where one would be more suspicious union might be needed. More information on this point is in [10].

Before leaving the subject of union, there is something to be learned from trying to give an example where union is needed. Suppose we let $S_{0}$ be the integers, and $S_{n+1}$ be the jump of $S_{n}$, that is, $S_{n+1}=\left\{e:\{e\}^{S_{n}}(e)\right.$ is defined $\}$. Let $S$ be $\left\{\langle e, n\rangle: e \in S_{n}\right\}$. What axioms are needed to form $S$ ? (Incidentally, although $S$ is natural enough in recursion theory, it is not part of "constructive mathematical practice" as defined above - it would not naturally occur in Bishop's book.) First, we would need to form the sequence $S_{n}$ (that is, the function $f(n)=S_{n}$. To do this, we would like to apply $D C$, but to do that, we need a set (of sets of integers) which is closed under the jump operation. For this we seem to need replacement, which is not part of $\boldsymbol{B}$. In $\boldsymbol{B}$, replacement has been replaced by abstraction, which does not suffice for the present example. Indeed, intuitively speaking, abstraction would suffice only if the $S_{n}$ 's were somehow uniformly defined, instead of inductively so as to have increasing complexity. But then, again intuitively, we would be dealing with a "pre-joined" family. Thus, the work which Friedman did in checking that abstraction suffices instead of replacement, is closely related to the fact that union is not needed.

We have thus arrived at the conclusion: Constructive mathemat- 
ical practice can be formalized in $B$ minus extensionality minus union. If this were a subtheory of Feferman's theory $F$, we would have shown that constructive mathematics can be formalized along Friedman's lines in $F$. But the $\Delta_{0}$ comprehension axiom is not a part of $F$. In formalizing mathematics along Friedman's lines, do we really need the whole $\Delta_{0}$ comprehension axiom, or will Feferman's elementary comprehension axiom suffice to prove the existence of those sets we actually need? The answer is, that the elementary comprehension axiom does suffices, as far as I can see; basically because we need sets only a few ranks above the integers. If the reader has some doubts on this point, perhaps they will be settled when he or she sees in the next section how the $\Delta_{0}$ comprehension axiom can be formally interpreted in $F$; and see also the Appendix.

Next we come to formalizing mathematics in $F$ along Feferman's lines. Here there is less to be said: Bishop's book can be almost transliterated into Feferman's system, treating reals, for instance, as sequences of rationals, given by an operation. Of course, $F$ includes more axioms about operations than are needed, but this need not concern us here. The same work as for Friedman's systems shows that restricted induction is enough, and that we do not need a unionlike axiom. ${ }^{2}$

Thus, we have associated to each assertion $A$ of informal mathematical practice, two formal sentences $A_{H}$ and $A_{S}$, formalizing $A$ along Friedman's lines and Feferman's lines, respectively. (The subscripts $H$ and $S$ are first initials of Friedman and Feferman.) The question to be considered now is, do we have

$$
F \vdash\left(A_{H} \longleftrightarrow A_{H}\right) \text { ? }
$$

Consider what is involved here: Feferman regards a function as an

${ }^{2}$ It is interesting to consider formalizing the construction of the sets $S_{n}$ in Feferman's systems. We can use the recursion theorem to define an $f$ such that $f(n)=S_{n}$, since for a certain elementary $\phi$, we have $f(n+1)=c_{\phi}(f(n))$. But, using only restricted induction, we probably cannot prove $\forall n \mathrm{Cl}(f(n))$, or even that $f(n)$ is defined for all $n$, so that we are essentially in the same position as in Friedman's system, of having no family to take the union of. This depends crucially on the fact that induction is restricted, which is not so in Friedman's theories, where the difficulty remains even with induction.

To make this point clearer, consider the axiom in Feferman's set-up that corresponds to union. This is the join axiom, which allows us to form $\{\langle x, y\rangle: y \in f(x)$ and $x \in A\}$ if we know $\forall x \in A \mathrm{Cl}(f(x))$. If we allow full induction, then the system with join is considerably stronger than the system without. One can use join, together with the recursion theorem as illustrated above, to prove the consistency of $F_{0}+$ full induction. Aczel [1] has identified the strength of $F_{0}+$ join + full induction (also known as $E M N+C A$ ) as $\Sigma_{1}^{1}-A C$, while $F_{0}+$ full induction has the same strength as the arithmetic comprehension axiom. This is in sharp contrast to the corresponding situation in intuitionistic set theory, where union is still conservative even if full induction is added (by the method of Lemma 1 of [3]). 
operation (or, in an inessential variation, as an operation paired with a domain and range); Friedman regards a function as its graph. Can the two be proved equivalent? One direction is easy; if $f$ is an operation, we can always form its graph,

$$
\operatorname{Gr}(f) \cong\{\langle x, y\rangle: \operatorname{App}(f, x, y)\},
$$

using the comprehension axiom of $F$. And of course we can find the graph of $f$ restricted to some domain. Now consider the other direction. Suppose $X$ is a classification such that $\forall x \in A \exists ! y \in B(\langle x, y) \in X)$. Can we find an operation $f$ such that $\forall x \in A(\langle x, f(x)\rangle \in X)$ ? Not generally, because the required axiom of choice is not part of $F$. (In fact, it is inconsistent with $F$, as Friedman pointed out, and also implies the law of the excluded middle in $B$.) See $\S 3$. So there is an obstacle to the proof that $A_{S} \leftrightarrow A_{H}$. However, if $A$ is a countable set (in the strong sense that it is the image of some invertible function defined on all integers), then the axiom of choice

$$
\left(\mathrm{AC}_{A}\right) \quad \forall x \in A \exists y \in B(\langle x, y\rangle \in X) \longrightarrow \exists f \in B^{A} \forall x \in A(\langle x, f(x)\rangle \in X)
$$

is a consequence of DC. At the risk of boring some readers we prove this: assuming the hypothesis of $\mathrm{AC}_{A}$, and assuming that $g$ enumerates $A$, we have

$$
\begin{aligned}
& \forall\langle n, a, b\rangle \in \omega \times A \times B \exists\langle m, x, y\rangle \\
& \quad \in \omega \times A \times B(m=n+1 \& x=g(m) \&\langle x, y\rangle \in X) .
\end{aligned}
$$

Also, there is $y$ in $B$ with $\langle g(0), y\rangle \in X$. Hence, by DC, there is some $F$ with $F(0)=\langle 0, g(0), y\rangle$ and for all $n, F(n)=\langle n, g(n), h(n)\rangle$, where $h$ is a function such that $\langle g(n), h(n)\rangle \in X$. Now we find the desired choice function $f$ in $F$ by $f(x)=h\left(g^{-1}(x)\right)$. Note that only restricted induction is needed to prove the above properties of $F$. Note also that we need the strong countability of $A$; it does not seem to work if we know only that $A$ is an image of the integers.

2. The arithmetic theorems of $B$ and $F$. In [11], Friedman proved that every arithmetic theorem of $B$ is already a theorem of classical arithmetic. Here we improve this result to show that $\underline{B}$ is conservative over intuitionistic arithmetic $H A$. This proof relies in part on the "Goodman-style" theorems of [2]; without this work, we still obtain a new proof of Friedman's theorem, which we believe is illuminating because of the light it casts on the fundamental concepts of such notions as "set" and "function" in constructive mathematics. In the process, we prove also that the arithmetic theorems of $F$ are also those of $H A$. (Feferman was the first to point out that the analog of Friedman's result holds for his system $B E M+C A$ with 
restricted induction, i.e., $F_{0}$ without $D C$. This result, though very easy to prove, seems to be foundationally significant.)

The four main steps of our proof have been outlined in the introduction, but now we want to give a more detailed discussion, before proceeding to the details of the proof. As discussed there, the first and last steps of the proof are the easiest, namely getting rid of extensionality, and showing $F_{0}$ is conservative over $H A$. The hard part is interpreting $\boldsymbol{B}$ minus extensionality in Feferman's theories. If we try to interpret it directly, we have the problem that $A C$ is inconsistent with $F_{0}$, but we seem to need $A C$ to interpret the exponentiation axiom by proving the equivalence of functions-asgraphs and functions-as-operations. The way out of this difficulty is found by first ignoring the difficulty: we give an interpretation of $\boldsymbol{B}$-ext in the language of Feferman's theory, by explaining what a "set" is in terms of the notion of "classification". This assigns to each formula $\phi$ of $\boldsymbol{B}$-ext, its interpretation $\phi^{0}$, in a natural and beautiful way. Unfortunately, we need $A C$ to prove the interpretation of the exponentiation axiom, and we need the full elementary comprehension axiom to prove the interpretation of the $\Delta_{0}$-separation axiom; and the combination is inconsistent. What to do?

Here is the answer. We find an axiom of choice, which we call $C A C$, which is sufficient to interpret the exponentiation axiom, but is consistent with $F$. We then interpret $B$-ext in $F+C A C$, using the explanation of "set" in terms of "classification." Then we show $C A C$ can be eliminated, along with $D C$, from proofs of arithmetic sentences.

We now define and discuss this new axiom $C A C$. We shall use $O P(A, B)$ for the set of operations from $A$ to $B$, instead of $B^{A}$, which might be ambiguous in the context of considering both functions and operations. Precisely, $O P(A, B)=\{f: \forall x \in A \exists y \in B \operatorname{App}(f, x, y)\}$. Now the new axiom is

$C A C: \quad \forall A \exists W\left(\forall w \in W\left((w)_{0} \in A\right) \quad \& \quad \forall x \in A \exists w \in W\left((w)_{0}=x\right) \&\right.$ $(\forall x \in A \exists y \in B(\langle x, y\rangle \in F)$

$\left.\left.\longrightarrow \exists f \in O P(W, B) \forall w \in W\left(\left\langle(w)_{0}, f(w)\right\rangle \in F\right)\right)\right)$.

The rationale for $C A C$ is that the definition of $A$ may involve some existential quantifiers which are "made explicit" in $W$; that is, $W$ consists of pairs $\langle x, z\rangle$ where $z$ codes the reasons why $x$ is in $A$. For instance, if $A$ is the set of continuous functions on an interval, then $W$ is the set of such functions together with moduli of continuity.

This axiom and its rationale should be carefully compared with the discussion of $A C$ ! in $\S 3$. The name $C A C$ stands for "careful axiom of choice", because we are careful not to think that $f(y)$ can 
be computed from $y$ in $A$ alone, but must also be computed from the information that allows us to know that $y$ is in $A .^{3}$

We now state our main theorem, and give the steps of the proof afterwards as a sequence of lemmas.

THEOREM 2.1. $F, B$, and $H A$ all have the same arithmetic theorems.

Proof. We establish the following chain of implications: Suppose $\phi$ is arithmetic, and $B$ proves $\phi$. Then

$$
\begin{array}{ll}
B-\operatorname{ext} \vdash \phi & (\text { Lemma } 1) \\
F+C A C \vdash \phi & (\text { Lemma } 2) \\
F_{0} \vdash \phi & (\text { Lemma } 5) \\
H A \vdash \dot{ } & (\text { Lemma } 6)
\end{array}
$$

Here come the lemmas:

LEMMA 1. Every arithmetical theorem of $\underline{B}$ can also be proved in $\underline{B}$ minus extensionality.

Proof. This is proved in [3].

LEMma 2. There is a natural interpretation of $\boldsymbol{B}$-ext in the language of Feferman's theories; if $\dot{\phi}^{0}$ denotes the interpretation of $\phi$, then every axiom $\phi$ of $\boldsymbol{B}$-ext except exponentiation has $\phi^{0}$ provable in $F$, and if $\phi$ is the exponentiation axiom, then $\phi^{0}$ is provable in $F+C A C$. If $\phi$ is arithmetic, $\phi^{0}$ and $\phi$ are equivalent in $F$.

Proof. Here we border on the foundational problem of explaining the different notions of set. We shall define in terms of "classification", a suitable notion of "set" such that the axioms of $\boldsymbol{B}$-ext

${ }^{3}$ In unpublished memoranda (to appear in expanded form with [10]), Feferman studies a system $C M$ in his language, which consists of $F_{0}^{-}$together with the schema $\phi \leftrightarrow$ $\exists x(x r \phi)$. It is not difficult to see that $C M$ proves $C A C$. Therefore $C M$ could be used in place of $F_{0}+C A C$ in $\S 2$, with some minor technical advantages. I choose to forego those advantages, because I wanted to interpret $\boldsymbol{B}$ in a "minimal" theory; that is, to exhibit a theory showing "just what is needed" to interpret $\boldsymbol{B}$. It seems important to do this in view of the fact that $I$ do not know how to interpret $\boldsymbol{B}$ in a theory which is constructively valid in a meaning-preserving way. Feferman makes a case that $C M$ should be considered constructively valid; that is, he describes an informal interpretation of the language under which $C M$ is valid. The fact that $C A C$ is valid on this interpretation is interesting, but there are also interpretations under which $C A C$ is not valid. So long as we lack a thorough analysis of the different possible constructive notions of "set", it seems worthwhile to explicitly exhibit the main principle needed to interpret $\boldsymbol{B}$. 
(except exponentiation) will be valid for "sets" (and this can be seen in $F$.) A "set" is a classification which "comes equipped" with certain auxiliary information. This auxiliary information should be essentially the structure where $y$ is the transitive closure of the set in question. This idea was exploited already in Lemma 1 and related results of [3]; but since that was written, we have found a neater way of presenting the "auxiliary information". We take as auxiliary information for $x$, the classification of all finite descending $\varepsilon$-chains beginning with $x$. (We use the pairing functions of $F_{0}$ to define codes of finite sequences in $F_{0}$; for these we use the usual number-theoretic notation, trusting no confusion will ensue. Thus $(x)_{0}, \cdots,(x)_{i}$, and $\operatorname{lh}(x)$ are given by certain application terms. Note that these are not the set-theoretic iterated ordered pairs. Note also that $(x)_{0}$ is $\boldsymbol{p}_{1}(x)$, an annoying difference in subscripts.) Let $S\left(x, x^{\prime}\right)$ be the formula,

$$
u \in x^{\prime} \longleftrightarrow(u)_{0}=x \quad \& \quad \forall i<\ln (u)-1\left((u)_{i+1} \in(u)_{i}\right) .
$$

Of course this is not an elementary formula, but no matter. We now interpret the sets of $B$-ext as pairs $(x, y)$ with $S(x, y)$. We interpret $a \in b$ as $(a)_{0} \in(b)_{0}$. To be consistent with this definition of membership, we interpret $N(x)$ as $(x)_{0} \in N$. After stating this so precisely, we shall ofter refer to $x$ as a "set" and $x$ ' as its "transitive closure", in case $S\left(x, x^{\prime}\right)$. We even speak of "the" transitive closure of $x$, when in the absence of extensionality we should say " $a$ " transitive closure. These abuses of terminology will cause no harm.

Now we turn to verifying the interpretations of the axioms.

Pairing. First, if $a$ and $b$ are sets (or even only classifications) then $\{a, b\}$ is a classification, by elementary comprehension. (Remark: this is true even in $F_{0}^{-}$, where disjunction is not allowed in the comprehension axiom; but we don't need this.) Furthermore if $S\left(a, a^{\prime}\right)$ and $S(b, b)^{\prime}$, then we can form the transitive closure $y$ of $\{a, b\}$ by putting $u \in y$ iff

$$
\begin{aligned}
(u)_{0}=\{a, b\} \text { and } \quad & \left(\left\langle(u)_{1}, \cdots(u)_{l h(u)-1}\right\rangle \in a^{\prime}\right. \\
& \left.\left\langle(u)_{1}, \cdots(u)_{l h(u)-1}\right\rangle \in b^{\prime}\right) .
\end{aligned}
$$

(And again, if we ever need it, the use of disjunction can be avoided.) Let $T C(x)$ abbreviate "transitive closure of $x$ ".

Union. Suppose $z$ is a set, i.e., $T C(z)$ exists. Then we must prove $\mathrm{U}_{x \in z} x$ is a set. First, why is it even a classification? We have $u \in \bigcup_{x \in z} x$ if $\exists x(x \in z$ and $u \in x)$, which is not an elementary formula: but we have $\bigcup_{x \in z} x=\left\{(p)_{2}: p \in T C(z)\right\}$, which solves the problem quite neatly. Thus the union of a set is a classification, although 
we cannot prove the union of a classification is a classification. Still, we have to prove that the union is a set, i.e., we have to construct its transitive closure. For this, we note that if $T C(z)$ exists, then for $w \in z, T C(w)$ is $\left\{\left\langle(u)_{1}, \cdots,(u)_{l h(u)}\right\rangle:(u)_{1}=w\right.$ and $\left.u \in T C(z)\right\}$. Thus $T C(w)$ is $c_{\phi}(T C(z))$ for a certain elementary $\phi$. Then we have

$$
\begin{array}{r}
w \in T C\left(\bigcup_{x \in z} x\right) \longleftrightarrow(w)_{0}=\bigcup_{x \in z} x \text { and }(w)_{1} \in z \text { and } \\
(w)_{2}, \cdots,(w)_{l h(w)} \in T C\left((w)_{1}\right) ;
\end{array}
$$

this last $T C\left((w)_{1}\right)$ is really $c_{\phi}(T C(z))$, so we have stayed within $F_{0}$.

Infinity. We have to prove that $N$ is a set;

$$
T C(N) \text { is just }\{\langle N, m\rangle: m \in N\} \text {. }
$$

$\Delta_{0}$-separation: We shall show that to every $\Delta_{0}$ formula $\phi$, with free set variables $x$ and $Y=Y_{1}, \cdots, Y_{n}$, and possibly some number variables not shown, there is an elementary formula $\phi^{\prime}$ such that $F_{0}$ proves

$$
\begin{aligned}
& S\left(A, A^{\prime}\right) \quad \& \quad S\left(Y, Y^{\prime}\right) \quad \& \quad S\left(x, X^{\prime}\right) \\
& \longrightarrow\left(x \in A \quad \& \quad \phi(x, Y) \longleftrightarrow \phi^{\prime}\left(x, A, A^{\prime}, Y, Y^{\prime}\right)\right) \text {. }
\end{aligned}
$$

Note that $X^{\prime}$ does not occur in $\phi^{\prime}$. We also have to prove a similar statement when $x$ is a number variable, replacing $S\left(x, X^{\prime}\right)$ by $x \in N$. (Some details: one has to give the definition of $\Delta_{0}$-formula of $\boldsymbol{B}$ in such a way that each variable is clearly a "set variable" or a "number variable"; actually there is only one sort of variables. Each formula of $\boldsymbol{B}$ makes sense as a formula of $F_{0}$, too. Even though we are thinking of $x$ as a set, we use a small variable to indicate it is not one of the classification parameters of $\phi^{\prime}$.)

If $\phi$ is atomic, it is either $x \in Y, Y \in x$, or $x=Y$. Here it is possible that $Y$ might be one of the constants of $B$, namely 0 or the constant for the successor function. In case $\phi$ is $x \in Y$ or $Y \in x$, we take $\phi^{\prime}$ to be $\phi$ and $x \in A$, which is already elementary, if $Y$ is a variable. If $Y$ is the successor function, then $(x \in Y)^{\prime}$ expresses that $x$ is a set-theoretic ordered pair $\left(m, m^{\prime}\right)$ for some integer $m ;(x=0)^{\prime}$ is $x=0$; finally $(x=C)^{\prime}$, where $C$ is the constant for the successor function, is given implicitly by our remark that formulae of $\boldsymbol{B}$ make sense in $F$; namely, it is $x=\left\{\left(n, n^{\prime}\right): n \in N\right\}$. This takes care of the basis cases in an inductive construction of $\phi^{\prime}$. Now suppose that $\phi$ is $\forall b \in z D(b, z, x, Y)$. Then, there is an elementary formula $D^{\prime}\left(b, b^{\prime}\right.$, $\left.z, z^{\prime}, Y, Y^{\prime}, x, A, A\right)$ such that $F$ proves $S\left(A, A^{\prime}\right) \& S\left(b, b^{\prime}\right) \& S\left(z, z^{\prime}\right)$ $\& S\left(Y, Y^{\prime}\right) \& S\left(x, x^{\prime}\right) \rightarrow D^{\prime}$. (Note, $D^{\prime}$ does not contain $x^{\prime}$.) We would 
like to set $\phi^{\prime}$ to be $\forall b\left(\langle z, b\rangle \in z^{\prime} \rightarrow D^{\prime}\right)$. This would not quite do, since $D^{\prime}$ contains a variable $b^{\prime}$ which is not allowed in $\phi^{\prime}$. We should substitute for this variable, $b^{\prime}=\left\{u:(u)_{0}=b \&\langle z\rangle * u \in z^{\prime}\right\}$, where $*$ denotes concatenation of sequences. This determines $\phi^{\prime}$ in this case. We leave the case of existential bounded quantification to the reader. If $\phi$ is $A \& \boldsymbol{B}$, then $\phi^{\prime}$ is $A^{\prime} \& \boldsymbol{B}^{\prime}$; if $\phi$ is $A \rightarrow \boldsymbol{B}$, then $\phi^{\prime}$ is $A^{\prime} \rightarrow \boldsymbol{B}^{\prime}$. The property which we claimed for $\phi^{\prime}$ is easily established by induction on the complexity of $\phi$.

Once we have $\phi^{\prime}$ in hand, it is easy to verify the $\Delta_{0}$-separation axiom. Namely, $\{x \in A: \phi(x)\}$ is defined by $\left\{x: \phi^{\prime}\left(x, A, X^{\prime}\right)\right\}$, not showing any additional parameters. Since $\phi^{\prime}$ is elementary, we can form this classification in $F_{0}$; call it $Q$. We have to construct the transitive closure of $Q$, too. But this is just $\left\{\langle Q\rangle * u:\langle A\rangle * u \in A^{\prime}\right.$ and $\left.(u)_{0} \in Q\right\}$. This verifies the interpretation of the $\Delta_{0}$-separation axiom.

Dependent choice: Suppose $S\left(A, A^{\prime}\right)$ and $S\left(X, X^{\prime}\right) \forall x \in A \exists y \in$ $A((x, y) \in X)$; here round brackets denote the set-theoretic ordered pair. Let $X_{0}$ be $\{\langle x, y\rangle:(x, y) \in X\}$; pointed brackets denote the pairing function in $F_{0}$. Then $\forall x \in A \exists y \in A\left(\langle x, y\rangle \in X_{0}\right)$. Hence, by $D C$ in $F$, there is for each $x \in A$, an operation $f$ from $N$ into $A$ such that $f(x)=0$, and $\forall n \in N\langle f(n), f\langle n+1)\rangle \in X_{0}$ Hence $\forall n \in N$ $(f(n), f(n+1)) \in X$. Let $g$ be $\{(u, v): u \in N$ and $\operatorname{App}(f, u, v)\}$. Then $g$ is the desired set-theoretic choice function. It is a classification; to see that it is a set, we have to define its transitive closure. Recalling that $(u, v)$ is just $\{\{u\},\{u, v\}\}$, this is easy to do, using

Abstraction. Suppose $A$ is a set, with $S\left(A, A^{\prime}\right)$. Then

$$
\{\{x \in A: \phi(x, y)\}: y \in A\},
$$

for a $\Delta_{0}$-formula $\phi$, can be formed by elementary comprehension, as $Q=\left\{\left\{x \in A: \phi^{\prime}\left(x, y, Y^{\prime}, A, A^{\prime}\right)\right\}: y \in A\right\}$, where $\phi^{\prime}$ is the formula constructed in verifying the separation axiom, and

$$
\left.Y^{\prime}=\left\{\langle w)_{1}, \cdots,(w)_{(h(w)-1}\right\rangle: w \in A^{\prime} \quad \text { and } \quad(w)_{1}=y\right\} .
$$

Moreover, the transitive closure of this set $Q$ can be defined by $Q^{\prime}=\left\{\langle Q, b\rangle * w: b \in Q \quad \&\left(w_{0}\right) \in b \quad \&\langle A\rangle * w \in A^{\prime}\right\}$.

Induction. The interpretation of the induction axiom of $\boldsymbol{B}$ is a consequence of the induction axiom of $F$, since every set is a classification. (Though possibly induction for sets is weaker than induction for classifications.)

We now turn to the exponentiation axiom. It will be helpful to see why we cannot interpret it directly. The exponentiation axiom says that if $A \& B$ are sets, then there is the set of all (graphs of) 
functions from $A$ to $B$. We can, of course, form the set $O P(A, B)$ in $F_{0}$. Let $\operatorname{Gr}(f, A)$ be $\{(x, y): x \in A \& \operatorname{App}(f, x, y)\}$; then let $X$ be $\{\operatorname{Gr}(f, A): f \in O P(A, B)\}$. So every member of $X$ is a function from $A$ to $B$. But to show every function from $A$ to $B$ (or some function with the same values) arises as $\operatorname{Gr}(f, A)$ for some operation $f$, we seem to need $A C$. Now here is how the axiom $C A C$ was discovered: giving up on proving the interpretation of the exponentiation axiom, I considered whether this interpretation was realized. (The reader unfamiliar with realizability can skip this part.) If $g$ is realized to be a function from $A$ to $B$, then there will be an operation $h$ such that whenever $u$ realizes $x \in A$, then $h(x, u)$ is $g(x)$. Hence $g$ can be recovered as $\{(x, y): \exists u(u r x \in A$ and $h(x, u) \cong y)\}$. Thus $A C$ would not be needed to prove the realizability interpretation of $\dot{\phi}^{0}$, where $\dot{\phi}$ is the axiom of exponentiation. Rather than carry the proof through along those lines, it seemed preferable to isolate $C A C$ and use it.

Now we use $C A C$ to verify $\dot{\phi}^{0}$, where $\phi$ is the exponentiation axiom. Let $A$ and $\boldsymbol{B}$ be sets. Find $W$ as in $C A C$. Form the classification $O P(W, \boldsymbol{B})$. Define $H(h)$, for $h$ in $O P(W, B)$, to be $\{(x, y)$ : $\exists b(\langle x, b\rangle \in W$ and $h(x, b) \cong y)\}$. This an be done with elementary comprehension. Now set $X=\{H(h): h \in O P(W, \boldsymbol{B})$ and $\forall x, b, c(\langle x, b\rangle \in W$ $\&\langle x, c\rangle \in W \rightarrow h(x, b)=h(x, c))\}$. This also can be done with elementary comprehension. Now we claim that $X$ is the set of functions from $A$ to $B$. First, if $f \in X$, then $f$ is (the graph of) a function from $A$ to $\boldsymbol{B}$, by the second clause in the definition of $X$. Secondly, if $F$ is a function from $A$ to $\boldsymbol{B}$, fix $W$ as in $C A C$; then $F$ arises as $H(h)$, where $h$ is the choice function given by $C A C$. (More precisely, a function with the same values as $F$ arises as $H(h)$, which is good enough.) Thus the set of functions from $A$ to $\boldsymbol{B}$ is a classification; but it is easy to define its transitive closure in terms of the transitive closures of $A$ and $\boldsymbol{B}$. Hence it is a set.

Finally, we need to say something about the assertion that $\dot{\phi}^{0}$ is equivalent to $\phi$ for $\dot{\phi}$ arithmetic. In $\boldsymbol{B}$, "arithmetic" sentences are built up from a constant for zero, a constant for successor, but no symbols for plus and times; while in $F_{0}$, there are application terms for plus, times, and successor. We have left implicit that in defining $\dot{\phi}^{0}$, zero should be interpreted as zero, and the successor should be interpreted as the graph of the successor function in $F_{0}$. We then prove in $F_{0}$ that the graphs of plus and times satisfy the interpretations of the defining formulae for plus and times in set theory (which say that plus and times satisfy certain recursion relations). We also prove that if any classification satisfies these defining formulae, then it is the graph of plus (or times, as the case may be). This takes care of the basis case of an easy induction on the complexity of arithmetic formulae $\phi$. This completes the proof of 
Lemma 2.

So far, we do not even know that $C A C$ is consistent. To prove this, and eventually to prove that $C A C$ can be eliminated from proofs of arithmetic sentences, we need realizability, which so far has not been discussed in this paper. We assume familiarity with the general idea of realizability. (See [13] for this.) In particular we need realizabilities for Fefermans's systems. There are two (main) kinds of realizability for Feferman's systems, which differ only on the definition of $e r x \in y$. The other clauses, which assign a formula $\operatorname{erA}$ to each formula $A$, are as follows:

$$
\begin{array}{lll}
\operatorname{er} A \& B & \text { is } & \left(e_{0}\right) r A \text { and }(e)_{1} r B \\
\operatorname{er} A \vee B & \text { is } \quad\left((e)_{0}=\longrightarrow(e)_{1} r A\right) \& \\
& \left((e)_{0} \neq 0 \longrightarrow(e)_{1} r B\right) \&(e)_{0} \in N \\
\operatorname{er}(A \longrightarrow B) & \text { is } \forall p(p r A \longrightarrow e(p) r B) \\
\operatorname{er} \exists x A & \text { is } \quad(e)_{1} r A\left((e)_{0}\right) \\
\operatorname{er} \forall x A & \text { is } \quad \forall x(e(x) r A) .
\end{array}
$$

Now if we take $e r x \in y$ to be $x \in y$, we get what is called " 1945 realizability for $F_{0}^{-}$. The name arises as follows: This realizability is similar to Kleene's original realizability, which has come to be known as "1945-realizability for $H A$ ", after the date of publication. This realizability is "for $F_{0}^{-}$", because with this definition of $e r x \in y$, we cannot get the full elementary comprehension axiom realized, but only the almost-negative elementary comprehension axiom of $F_{0}^{-}$. (However, we do get $A C$ realized; set §3.) In order to make up a realizability for $F$, we have to take $e r x \in y$ to be $\langle e, x\rangle \in y$. It is this realizability which we shall use.

Realizability for $F_{0}^{-}$is in [9] (though there is an oversight in the treatment of disjunction). Realizability for $F_{0}$ (and stronger theories) is in [4]. There it is given with the " $x *$-trick", in which we make a variable $x^{*}$ correspond to each variable $x$, and then the free variables of $e r A$ are $e$ together with $x^{*}$ for $x$ free in $A$. This is necessary if we are doing a variant kind of realizability called " $q$-realizability", but it is not necessary for 1945-realizability. Nevertheless, it is often a convenient aid, because the meaning of $x$ in $\operatorname{er} A(x)$ is not the same as it is in $A(x)$, when $e r x \in y$ is taken as $\langle e, x\rangle \in y$. So one think of $x^{*}$ as the set of $\left\langle e, y^{*}\right\rangle$ such that $e$ realizes $y \in x$. On the other hand, we want $\operatorname{er} \operatorname{App}(f, x, y)$ to be $\operatorname{App}(f, x, y)$, and if we use the $x^{*}$ notation, it has to be $\operatorname{App}\left(f^{*}, x^{*}, y^{*}\right)$, which is an annoyance. It is, however, possible to have the best of both notations: just let $x^{*}$ be $x$ itself. This is what we do. We define $e r \mathrm{Cl}(x)$ to be $\mathrm{Cl}(x)$. 


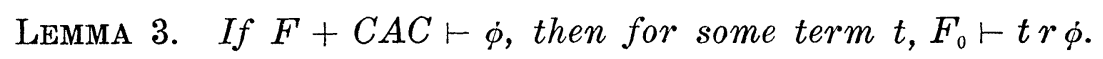

Corollary. F+CAC is consistent.

Proof of lemma. By induction on the length of proof of $\phi$. The axioms of $F_{0}$ have been dealt with in [4]. Actually, when [4] was written, the importance of restricted induction had not yet been realized, so one has to add the observation that restricted induction suffices to prove the realizability of restricted induction. The axiom of dependent choices is realized; we leave the verification of this to the reader. The main task which confronts us here is to prove that $C A C$ is realized. We now give the argument for this. Suppose $A^{*}$ is given. Let $W^{*}=V \times\left\{\langle u, v\rangle:\langle v, u\rangle \in A^{*}\right\}$, so that $e r\langle x, u\rangle \in W$ iff $\langle e,\langle x, u\rangle\rangle \in W^{*}$ iff $u r x \in A$. Then $\forall w \in W\left((w)_{0} \in A\right)$ is realized, since if erw $w$, then $(w)_{1} r(w)_{0} \in A$. Also $\forall x \in A \exists w \in W\left((w)_{0}=x\right)$ is realized, since if $u r x \in A$, then $\langle x, u\rangle$ is realized to be in $W$. Suppose $e r \forall x \in A \exists y \in B(\langle x, y\rangle \in F)$. Then, if $u r x \in A$ (that is, if $e r\langle x, u\rangle \in W$ for some $e)$ we have $(e(x, u))_{0}$ is $y$ and $(e(x, u))_{1}$ is $p$ such that $(p)_{0} r y \in \boldsymbol{B}$. Take $f=\lambda w(e(w))_{0}$. We claim $f \in O P(W, \boldsymbol{B})$ is realized; that is, $\forall w \in W \exists y \in \boldsymbol{B} \operatorname{App}(f, w, y)$ is realized. Suppose e $r w \in W$; Then (with $w=\langle x, u\rangle$ as above) we have that $f(w)=$ $(e(w))_{0}$ is $y$ and $(e(w))_{1}$ is $p$ such that $(p)_{0} r y \in B$; \& $\operatorname{App}(f, w, y)$ is realized, being true. Finally we claim $\forall w \in W\left(\left\langle(w)_{0}, f(w)\right\rangle \in F\right)$ is realized. Suppose $e r w \in W$; let $w=\langle x, u\rangle$; then $f(w)$ is $y$ and $(e(w))_{1}$ is $p$ such that $(p)_{1} r\langle x, y\rangle \in F$, by our hypothesis that $e r \forall x \in A \exists y \in$ $\boldsymbol{B}(\langle x, y\rangle \in F)$. But this is just what we had to show. Furthermore, all the realizing numbers we have produced depend uniformly on the realizers of the various hypotheses. This completes the proof of the lemma.

Lemma 4. Suppose $\phi$ is arithmetic and $F+C A C \vdash \phi$. Then $F_{0}+$ classical logic $\vdash \phi$.

REMARK. This lemma is not needed for the proof of our main theorem, but is included only to show how a simple proof of Friedman's theorem that $\boldsymbol{B}$ is conservative over classical arithmetic can be obtained by these methods.

Proof. Let $\phi$ be fixed. Then let $A$ be a set encoding the characteristic functions of all the (finitely many) subformulae of $\phi$, so that each of them is recursive in $A$, and $A$ is arithmetic. We can use functions recursive in $A$ for a formalized realizability, and by standard methods (see, e.g., [13, p. 193] or the first part of [2]), we can show that every subformula $\Psi$ of $\phi$ is "self-realizing" in the 
sense that there is a term $t_{\Psi}$ such that $(\operatorname{er} \Psi \rightarrow \Psi)$ and $\left(\Psi \rightarrow t_{\Psi} r \Psi\right)$ are provable in $F_{0}$ plus classical logic (one needs classical logic for reasons discussed in the first part of [2]). By Lemma $3, F_{0} \vdash t r \phi$ for some term $t$. Hence, $F_{0}$ plus classical logic proves $\phi$.

Lemma 5. If $F+C A C \vdash \phi$, then $F_{0} \vdash \phi$, for arithmetic $\phi$.

Proof. This is a Goodman-style conservative extension result of the sort considered in [2]. In that paper (Theorem 6.3), it is shown that any axiom of choice can be eliminated, as long as it is provably realized. Since we have shown $C A C$ is provably realized, the proof of Theorem 6.3 of [2] yields the lemma.

Lemma $6 . \quad F_{0}$ is conservative over $H A$, and $F_{0}+$ classical logic is conservative over classical arithmetic $P A$.

REMARK. The second statement, together with Lemma 4, yields a proof of Friedman's theorem independent of [2]. The second statement of the lemma is due to Feferman (unpublished).

Proof. First we prove the second statement. If $\phi$ is not provable in $P A$, there is a model $\mathscr{N}$ of $P A$ in which $\phi$ fails. Then, applying the basic technique of [9] for constructing models of $F_{0}$, we get a model of $F_{0}$ with universe $\mathscr{N}$, in which $\operatorname{App}(e, x, y)$ holds just in case $\mathscr{N} \vDash \exists n(T(e, x, n) \& U(n)=y)$; the integers of this model $\mathscr{C}$ are recursively isomorphic (in $\mathscr{N}$ ) to the integers of $\mathscr{N}$, so the same arithmetic sentences $\phi$ hold in $\mathscr{C}$ as in $\mathscr{N}$. Hence, $\phi$ also fails ia $\mathscr{l}$, proving the second part of the lemma.

For the sake of completeness, and for other reasons, we review Feferman's model construction used above. The other reasons are: to check that we can start with a nonstandard model; to furnish some ground for generalizing the construction below; and to simplify the exposition, since in [9] Feferman is modeling stronger axioms, which makes the construction more complicated than it needs to be here.

Given a classical model $\mathscr{N}$, then interpret $\operatorname{App}(f, x, y)$ as $\mathscr{N} F$ $\exists n(T(f, x, n) \& U(n)=y)$. The combinatorial constants have natural interpretations as certain (standard) integers. The integers of $\mathscr{K}$ will be generated using the "successor" function $\langle x, 0\rangle$ from 0 ; but generated inside $\mathscr{N}$. That is, we define $f$ by $f(0)=0, f(n+1)=$ $\langle f(n), 0\rangle ; f$ is actually $\{\mathrm{e}\}$ for some $\mathrm{c}$, and the integers of $\mathscr{C}$ are those elements of $\mathscr{N}$ which are satisfied to be in the range of $\{\mathrm{e}\}$. Then one defines relations $\mathbf{C l}$ and $\varepsilon$ on $\mathscr{N}$ to produce. $/ 4$. These 
are defined as the union of relations $\mathbf{C l}_{n}$ and $\varepsilon_{n}$, over all integers $n$. (In [9], a transfinite union is needed to deal with stronger axioms.) We can take $\mathbf{C l}_{0}$ and $\varepsilon_{0}$ to be empty, and set

$$
\begin{array}{r}
\mathscr{H} \vDash x \varepsilon_{n+1} c_{\phi}(y, W) \text { iff } W \in \mathbf{C l}_{n} \text { and } \mathscr{C} \vDash \phi(x, y, W) \\
W \in \mathbf{C l}_{n+1} \text { iff } W \text { is }\left\{\boldsymbol{c}_{\phi}\right\}(y, Z) \text { for } Z \text { in } \mathbf{C l}_{n} \text {, or } W \in \mathbf{C l}_{n} .
\end{array}
$$

Here $c_{\phi}$ is a number used to interpret the constant $c_{\phi}$; it can be the index of some trivial function, for instance $\left\{c_{\phi}\right\}(y, Z)=\left\langle 1,{ }^{\prime} \phi ', y, z\right\rangle$, remembering that $y$ and $Z$ can be lists of several variables, of the proper length to be substituted in $\phi$. This definition appears to be circular, but it is not, because $\phi$ is an elementary formula. See [9] for a more complete discussion. The point to be made here is that, for $F_{0}$, the same construction works over a nonstandard model $\mathscr{N}$, because we have only restricted induction. Since the integers of $\mathscr{M}$ are isomorphic to those of $\mathscr{N}$, in $\mathscr{N}$, and since $\mathscr{M} \vDash \phi(m)$ is expressed by a formula of arithmetic, for each $\phi$ of $F_{0}$, induction in $\mathscr{N}$ can be used to verify induction in $\mathscr{l l}$.

Now we turn to the proof of the first part of the lemma. The idea is to repeat the above construction using Kripke models. Let $\phi$ be an arithmetic sentence underivable in $H A$. According to Kripke's completeness theorem [13, p. 329], we can find a Kripke model $\mathscr{N}$ of $H A$ in which $\phi$ fails. Now, imitating Feferman's construction of a model for $F_{0}$, we "blow up" $\mathscr{N}$ to a Kripke model $\mathscr{N}$ of $F_{0}$. Furthermore, we arrange that $\mathscr{N}$ and $\mathscr{M}$ have isomorphic integers at each node, so that $\phi$ will also fail in $\mathscr{C}$. Hence $\phi$ is not a theorem of $F_{0}$.

The construction is straightforward: the domain $M_{t}$ of $\mathscr{M}$ at the node $t$ is just $N_{t}$, the domain of $\mathscr{N}$ at node $t$. Now the atomic relations $\varepsilon$ and $\mathrm{Cl}$ are defined (at each node $t$ ) as the union of relations $\mathrm{Cl}_{n}$ and $\varepsilon_{n}$, by the same clauses as in Lemma 4, reading them as holding at node $t$. But there is a little more to check. Why is this a "monotone" assignment? In other words, if $x \varepsilon y$ holds at $t$, and $t \leqq s$, why does $x \varepsilon y$ hold at $s$ ? Well, to prove this we have to prove by a double induction on $n$ and the complexity of $\phi$ that for $x, y$ in $\mathbf{C l}_{n}, t \leqq s$ and $t \vDash \phi(x, y)$ implies $s \vDash \phi(x, y)$. Of course this is known for Kripke models, but we have not yet constructed a Kripke model. The proof is straightforward, using that $\phi$ is elementary. The verification of the comprehension axiom and other axioms of $F$ is also straightforward. The final point concerns the arithmetic sentences. Note that the application relation is the same in all nodes of $\mathscr{M}$; in particular, the integers at each node are isomorphic to the integers of $\mathscr{N}$. It is then easy to prove by induction on the complexity of the arithmetic formula $A$ that for 
all nodes $t, \mathscr{M} \vDash(\boldsymbol{y})$ iff $\mathscr{N} \vDash A(y)$, where as before $\boldsymbol{y}$ is the element of $\mathscr{C}$ representing the integer $y$ of $\mathscr{N}$. This completes the proof, both of the lemma, and of the main theorem.

3. More on the axiom of choice. The strongest axiom of choice which I am willing to assert is constructively valid is $D C$. There are, however, people who argue for the validity of certain stronger forms. We now give a list of various forms of the axiom of choice which can be considered.

$A C_{X}: \quad \forall a \in X \exists b \in Y \phi(a, b) \rightarrow \exists f \in Y^{X} \forall a \in \phi(a, f(a))$. (Here $X$ can be a term, or a formula, depending on the context. $Y$ is a variable.)

$A C: \quad$ Like $A C_{X}$, but $X$ is a variable.

$A C_{F T}$ : Schema of instances of $A C$ in which both $X$ and $Y$ are finite types. (Here $w$ is a finite type, and if $A$ and $B$ are finite types, so is $A^{B}$.)

$A C$ ! and $A C_{X}$ !: like $A C$ and $A C_{X}$, but beginning with $\forall a \in X \exists ! b \in$ $Y \phi(a, b)$.

$A C^{0}: \quad \forall a \in X \exists b \phi(a, b) \rightarrow \exists f \forall a \in X \phi(a, b)$.

$A C_{X}^{0}$ : Like $A C^{0}$, but $X$ is given by a term or formula.

We now discuss these briefly. First, $A C^{0}$ follows in set theory from $A C$ and collection. It was introduced by Feferman, in [9]. The other versions are much older. We proved in $\S 1$ the simple result that $A C_{X}$ for $X$ countable follows from $D C$, so is constructively valid. A case can be made that $A C$ ! is constructively valid (Myhill calls it a principle of "nonchoice" in [12]); I now state this case to the best of my understanding. Suppose $\forall a \in X \exists ! b \in Y \phi(a, b)$ is valid. Then we have a method $h$ for going from an object $a$ and a proof $p$ that $a \in X$ to an object $h(a, p)$ such that $\phi(a, h(a, p)$ ) (and a proof of this fact). But because $\exists ! b \phi(a, b)$, we have $h(a, p)$ depending only on $a$, that is, if $p$ and $q$ are two different proofs that $a \in X$, then $h(a, p)=h(a, q)$. "Hence", say some people, there is a function $f(a)$ as required. This inference requires that each member of $X$ "come equipped" with a proof that it is a member of $X$; which is in fact often assumed in informal constructive mathematics. It seems to me, however, that there are several distinct notions of "set", and only on the strictest notion do we have the required property that members of $X$ come equipped with proofs that they are in $X$. Thus we may assert that $A C$ ! is valid, only for this notion of "set"; while $D C$ is valid generally, with specifying clearly our notion of set. These various notions of set have yet to be clearly analyzed. The following theorem summarizes the principal consistency results about 
these axioms of choice. ${ }^{4}$

THEOREM 3.1. (i) (Friedman) AC! is inconsistent with $F_{0}$.

(ii) (Friedman, announced in [12]) B $+A C$ ! $+C T$ is consistent.

(iii) $A C_{F T}+C T$ is consistent with Feferman's theories.

(iv) $\boldsymbol{B}+A C$ is of course classically true; but $\boldsymbol{B}+A C$ implies the law of the excluded middle for bounded formulae (Myhill, announced in [12]; and Dianesco (unpublished)).

This is due to the presence of extensionality. In fact

(v) (Troelstra) $C T+$ extensionality is inconsistent with $A C_{F T}$ (even $A C_{10}$ ) in $H A^{\omega}$. (It is an open problem whether $B$-ext $+A C+$ $C T$ is consistent.)

(vi) (Feferman) $F_{0}^{-}+A C+C T$ is consistent.

Proof. Here is Friĕdman's proof of (i): Let $A(x, y)$ be $(y=1$ and $\operatorname{App}(x, x, 0)) \vee(y=0$ and $\neg \operatorname{App}(x, x, 0))$. Let $X$ be $\{x: \exists y A(x, y)\}$. Then $\forall x \in X \exists ! y \in N A(x, y)$. Suppose there is a choice function $f$. Then $\neg \operatorname{App}(f, f, 0)$, since if $\operatorname{App}(f, f, 0)$, the only possible $y$ such that $A(f, y)$ is $y=1$, but $f(f)$ is such a $y$. But then the only possible $y$ such that $A(x, y)$ is $y=0$, and moreover $f \in X$ since $\neg \operatorname{App}(f, f, 0)$; hence $f(f)=0$, contradiction.

(ii) follows from the methods of this paper, because $\boldsymbol{B}+A C$ ! is interpreted in $F+C A C$, which is consistent with $C T$ by realizability. I do not know what proof Friedman had in mind - neither the realizability of [3] nor that of [12] will yield this result.

(iii) is proved in [4]. Generally speaking, one can get $A C_{X}$ consistent with Feferman's theories if $X$ is some particular selfrealizing set. But, for instance, can one get $A C_{X}$ consistent with $F_{0}$ if $X$ is defined by an almost-negative formula? One cannot seem to show that almost-negative formulae are self-realizing for the notion of realizability needed for $F_{0}$. For $F_{0}^{-}$, on the other hand, one has a simpler notion of realizability, and here $A C$ is realized. Hence (vi) is proved.

As to (iv), I do not know what proof Myhill had in mind. Here is a proof which D. Scott sent me on a postcard, and which is apparently due to Dianesco. Let $\phi$ be a given formula; we shall show $A C$ plus extensionality proves $\phi \vee \neg \phi$. Let $A$ be $\{n: n=0 \vee$ $(n=1 \& \phi)\}$ and $B$ be $\{n: n=1 \vee(n=0 \& \phi)\}$. Thus $\forall x \in\{A, B\} \exists y \in$

${ }^{4}$ Added in proof: A more careful distinction between the notions of operation and function will also help clarify these issues. While AC! is invalid for operations it is valid for functions, if these concepts are suitably formulated. 
$N(y \in x)$. Suppose $f$ is a choice function. We have $f(A)=f(B) \vee$ $f(A) \neq f(B)$. If $f(A)=f(B)$ then $\phi$, so $\phi \vee \neg \phi$. If $f(A) \neq f(B)$, then $\neg \phi$ can be derived: suppose $\phi$. Then $A=B$ by extensionality, so $f(A)=f(B)$, contradiction. Hence in either case $\phi \vee-\gamma$.

Now we prove (v) according to Troelstra: Suppose CT. Then there is, by $A C_{1,0}$, a functional $H$ such that $H(f)$ is an index $e$ such that $\forall x(f(x)=\{e\}(x))$. Let $g_{x}$ be defined by

$$
g_{x}(y)= \begin{cases}0 & \text { if } T(x, x, y) \\ 1 & \text { otherwise }\end{cases}
$$

Let

$$
h(x)= \begin{cases}0 & \text { if } H\left(g_{x}\right)=H(c) \text { where }\{c\} \text { is identically } 0 \\ 1 & \text { otherwise }\end{cases}
$$

By extensionality, $h(x)=0$ if and only if $g_{x}$ is identically 0 , which is if and only if $\{x\}(x)$ is undefined. Hence $h$ is not recursive, contradicting $C T$.

REMARKS. The reasons why $A C$ is too strong are quite different in Friedman's and Feferman's theories. Part (i) of the theorem shows that Feferman's axioms are not valid for every possible notion of "set"; in particular, not for any notion of set for which an element of $X$ "comes equipped" with a proof that it is an element of $X$. I wonder, in this connection, if $S\left(X, X^{\prime}\right) \rightarrow A C_{X}$ is consistent with $F_{0}$, where $S\left(X, X^{\prime}\right)$ is defined in proof of Lemma 2 of $\S 2$.

In a footnote to [4], I considered a version $C T_{2}$ of Church's thesis applying to partial functions, and showed that $C T$ does not imply $C T_{2}$. Now I add that $C T_{2}$ contradicts $A C$ ! in $\underline{B}$, while $C T$ does not. The contradiction is obtained by imitating the proof of (i) above.

APPENDIX. We intend to present here evidence that $\left(A_{H} \leftrightarrow A_{S}\right)$ holds for "naturally arising" assertions $A$ of mathematical practice. We have already considered the difficulties of proving this equivalence in general; the situation is unsettling, because if the two formalizations are as "natural" as it might seem, then they should both be equivalent to $A$ itself. The aim of this appendix is to show that, whatever the disagreement between the two formalizations, it does not manifest itself in the domain of mathematical practice. Originally we viewed this work as a "reconciliation" of the two approaches; but it became clear that giving equivalent results for mathematical practice is not sufficient for a reconciliation of two approaches so different in spirit. We must await a deeper philosophical analysis 
of the foundations of constructivism to explain more clearly the meaning of an informal assertion $A$, before we can decide whether $A_{H}$ or $A_{S}$ is closer to this meaning.

Let us now state more precisely the point:

Thesis. For each $A$ of informal constructive mathematical practice, perhaps excluding measure theory, $F \vdash\left(A_{S} \leftrightarrow A_{H}\right)$.

Of course, since the concept of mathematical practice is not rigorously defined, we cannot make this into a theorem and prove it. We propose to give evidence for it, by showing that in mathematical practice, the only functions which arise are defined on countable or finite sets, and so one can use the countable axiom of choice $A C_{N}$ to pass back and forth between Feferman's formalization of these functions, and Friedman's formalization.

To begin with, a real is a function from the integers to the rationals, so $A C_{N}$ suffices to prove the equivalence of Friedman's reals and Feferman's real. Now consider functions from the reals to the reals. Of course, the reals are not countable, so we cannot show that Friedman's $R^{R}$ corresponds to Feferman's $R^{R}$. The point here is that $R^{R}$ is not an object which arises in mathematical practice. In practice, we consider only continuous functions from $R$ to $R$; to be be precise, we consider the functions $C(R, R)$ which come equipped with a modulus of uniform continuity for each compact subset of $R$. The modulus of continuity may be considered as a function from rationals to rationals, producing $\varepsilon$ from $\delta$, good on $[n, n]$. Such a function $f$ is known once we know its modulus of continuity and its values on the rationals; hence, it is given by functions with countable domains. Hence, $A C_{N}$ applies to prove that Friedman's $C(R, R)$ and Feferman's $C(R, R)$ correspond. Now, the same method can be applied to $C(S, Y)$, if $S$ is a separable metric space. The values of $f$ are determined by its values on the countable base, together with the modulus of continuity, which is determined by its values on the rationals. Thus $F \vdash\left(A_{H} \leftrightarrow A_{S}\right)$ for each assertion $A$ about separable metric spaces. But this already encompasses all of Bishop's book, except perhaps the chapters on measure theory. (These chapters on measure theory are anyway superseded by the Bishop-Cheng notes, [8].)

Even if our thesis had to be restricted to that part of analysis not using measure theory, it is still significant. It is nevertheless worthwhile to consider the problems involved in extending the thesis. For instance, in the case of Lebesgue measure on the reals, as developed in [8], we start with a sequence of continuous functions $f_{n}$ such that $\Sigma \int_{0}^{1}\left|f_{n}(x)\right| d x$ converges. If $f(x)=\Sigma f_{n}(x)$ whenever this 
converges absolutely, then $f$ is called an integrable function. In order to extend our thesis to measure theory, we would have to prove some theorems about the domains of integrable functions, showing essentially that countably many values determine the function. While it is an interesting project to work such theorems out, I have not done so, because I believe they are somewhat tangential to the foundational issues involved. What is really needed here is the axiom of choice in the form $\forall x \in D \exists ! y \in R\langle x, y\rangle \in W \rightarrow \exists f \in$ $R^{D} \forall x \in D\langle x, f(x)\rangle \in W$, where $D$ is the domain of an integrable function. The definitive resolution of this issue will rest not on a special, technical analysis, but on a general analysis of constructive notions of set, justifying certain axioms of choice for certain kinds of sets. Such an analysis is beyond the scope of this paper.

\section{REFERENCES}

1. P. Aczel, unpublished material.

2. M. Beeson, Goodman's theorem and beyond, Pacific J. Math., 84, (1979), 1-16.

3. - Continuity in intuitionistic set theories, to appear in Proceedings of the Logic Colloquium 1978, North-Holland, 1979, ed. by D. van Dalen, M. Boffa and K. McAloon.

4. - Principles of continuous choice and continuity of functions in formal systems for constructive mathematics, Annals of Math. Logic, 12 (1977), 249-322.

5. — A type-free Gödel interpretation, J. Symbolic Logic, 43 (1978), 213-227.

6 . - Some relations between classical and constructive mathematics, J. Symbolic Logic, 43 (1978), 228-246.

7. E. Bishop, Foundations of Constructive Analysis, McGraw-Hill, 1967.

8. E. Bishop and H. Cheng, Constructive measure theory, Memoirs of the Amer. Math. Soc., No. 116, 1972.

9. S. Feferman, A language and axioms for explicit mathematics, in Algebra and Logic, Springer Lecture Notes No. 450, 87-139.

10. - Notes on the formalization of Bishop's constructive mathematics, mimeographed notes, Stanford University. These notes were subsumed in Constructive Theories of Functions and Classes, in Logic Colloquium '78, ed. by M. Boffa, D. van Dolen and K. McAloon.

11. H. Friedman, Set-theoretic foundations of constructive analysis, Annals of Math., 105 (1977), 1-28.

12. J. Myhill, Constructive set theory, J. Symbolic Logic, 40 (1975), 347-382.

13. A. S. Troelstra, Metamathematical investigations of intuitionistic arithmetic and arithmetic of finite types, Springer Lecture Notes No. 344.

Received March 30, 1977.

InSTITUTE FÜr Mathematik

Der UNIVERSITÄT BONN

WEGELERSTRASSE 6

53 Bonn, Germany 


\section{PACIFIC JOURNAL OF MATHEMATICS}

\section{EDITORS}

DONALD BABBITT (Managing Editor)

University of Galifornia

Los Angeles, California 90024

Hugo Rossi

University of Utah

Salt Lake City, UT 84112

C. C. MOORE AND ANDREW OGG

University of California

Berkeley, CA 94720
J. DugundjI

Department of Mathematics University of Southern California Los Angeles, California 90007

R. Finn and J. Milgram Stanford University Stanford, California 94305

ASSOCIATE EDITORS

E. F. BECKENBACH

B. H. NeumanN

F. WOLF

K. YoSHIDA

\section{SUPPORTING INSTITUTIONS}

UNIVERSITY OF BRITISH COLUMBIA

UNIVERSITY OF SOUTHERN CALIFONIA

CALIFORNIA INSTITUTE OF TECHNOLOGY

UNIVERSITY OF CALIFORNIA

MONTANA STATE UNIVERSITY

STANFORD UNIVERSITY

UNIVERSITY OF HAWAII

UNIVERSITY OF NEVADA, RENO

UNIVERSITY OF TOKYO

NEW MEXICO STATE UNIVERSITY

UNIVERSITY OF UTAH

OREGON STATE UNIVERSITY

WASHINGTON STATE UNIVERSITY

UNIVERSITY OF OREGON

UNIVERSITY OF WASHINGTON 


\section{Pacific Journal of Mathematics \\ Vol. 88, No. $1 \quad$ March, 1980}

Michael James Beeson, Extensionality and choice in constructive mathematics .................................... 1

José L. Blasco Olcina, Two questions on Wallman rings.............. 29

Peter I. Booth and J. Tillotson, Monoidal closed, Cartesian closed and convenient categories of topological spaces .................. 35

Peter B. Borwein, Rational functions with positive coefficients, polynomials and uniform approximations ......................... 55

Josip Globevnik, Fourier coefficients of the Rudin-Carleson extensions . . . . 69

Jacob Kofner, Quasimetrizable spaces ...................... 81

Mark Mandelker, Resolutions on the line ..................... 91

Lynn McLinden, An analogue of Moreau's proximation theorem, with application to the nonlinear complementarity problem ............ 101

Atsushi Murase, On the uniform distribution property of certain linear algebraic groups ................................ 163

Nicholas Th. Varopoulos, Zeros of $H^{p}$ functions in several complex

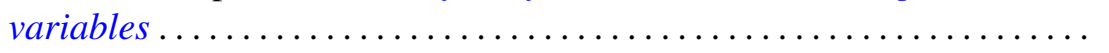

